\title{
LATTICE ISOMORPHISMS OF MODULAR INVERSE SEMIGROUPS
}

\author{
by K. G. JOHNSTON
}

(Received 29th January 1987)

\section{Introduction}

For an inverse semigroup $S$ we will consider the lattice of inverse subsemigroups of $S$, denoted $L(S)$. A major problem in algebra has been that of finding to what extent an algebra is determined by its lattice of subalgebras. (See, for example, the survey article [9]). By a lattice isomorphism ( $L$-isomorphism, structural isomorphism, or projectivity) of an inverse semigroup $S$ onto another $T$ we shall mean an isomorphism $\Phi$ of $L(S)$ onto $L(T)$. A mapping $\phi$ from $S$ to $T$ is said to induce $\Phi$ if $A \Phi=A \phi$ for all $A$ in $L(S)$. We say that $S$ is strongly determined by $L(S)$ if every lattice isomorphism of $S$ onto $T$ is induced by an isomorphism of $S$ onto $T$.

In previous papers, $P$. R. Jones and the author have investigated the structure of $L F(S)$, the lattice of full inverse subsemigroups of $S[2,3,4]$. We call $S$ modular [distributive] if $L F(S)$ is. In [5] Jones showed that any simple distributive inverse semigroup $S$ is strongly determined by $L(S)$. Indeed, each lattice isomorphism is induced by a unique isomorphism of $S$. Here we will extend this result and show that any simple modular inverse semigroup $S$ is strongly determined by $L(S)$ and is induced by a unique isomorphism.

Throughout this paper we will assume that $S$ and $T$ are inverse semigroups with semilattices of idempotents $E_{S}$ and $E_{T}$ respectively, and $\Phi$ an $L$-isomorphism of $S$ onto $T$. For basic properties of inverse semigroups the reader is referred to $[\mathbf{1 , 8}]$. If $(P, \leqq)$ is a poset, we write $p \| q$ if $p$ and $q$ are incomparable, and $p N q$ otherwise. We now state a preliminary result.

Result 1 ([6, Theorem 2.1]). Let $S$ be a simple inverse semigroup. If $L(S) \simeq L(T)$ then $T$ is also simple. Furthermore, $E_{S} \simeq E_{T}$ under the mapping $\phi_{E}: E_{S} \rightarrow E_{T}$ given by $\{e\} \Phi=$ $\left\{e \phi_{E}\right\}$ for all $e \in E_{S}$.

\section{Simple modular inverse semigroups}

We now consider the lattice $L F$, and basic facts about modular inverse semigroups needed later. An element $x$ of $S$ is called strictly right (left) regular if $x x^{-1}>x^{-1} x\left[x^{-1} x>x x^{-1}\right]$. Such an element generates a bicyclic subsemigroup. $E_{S}$ is Archimedean in $S$ if for any $e \in E_{S}$ and strictly right regular element $x, x^{-n} x^{n} \leqq e$ for some positive integer $n$. We let $K_{S}=\operatorname{ker} \sigma$, where $\sigma$ is the minimum group congruence on $S$. 
Result 2 ([2]). A simple inverse semigroup $S$ which is not a group is modular if and only if

(i) $S$ is combinatorial,

(ii) $E_{S}$ is Archimedean in $S$,

(iii) $S / \sigma$ is locally cyclic, and

(iv) the poset of idempotents of each D-class of $S$ is either a chain or contains exactly one pair of incomparable elements, each of which is maximal.

In the proof that simple distributive inverse semigroups are strongly determined by their lattice isomorphisms, much use was made of the fact that these semigroups are $E$ unitary, and hence $K_{S}=E_{S}$. This is not necessarily true in the modular case, so the kernel of $\sigma, K_{S}$, will be of interest.

Result 3 ([2, Propositions 3.1 and 4.2]). If $S$ is a simple modular inverse semigroup which is not a group, then $K_{S}=\left\{x: x x^{-1} \| x^{-1} x\right\} \cup E_{S}=\left\{x: x^{2}=x^{3}\right\}$, and for each $e \in E_{S}$, $\left|K_{S} \cap R_{e}\right| \leqq 2$.

If $S$ is a simple modular inverse semigroup which is not a group and $\Phi$ a lattice isomorphism of $S$ onto $T$, then by Result $1, E_{S} \Phi=E_{T}$. Thus $\Phi$ restricts naturally to an isomorphism of $L F(S)$ onto $L F(T)$, and therefore $T$ cannot be a group. $T$ must therefore also be a modular inverse semigroup, not a group, which is simple by Result 1 , meaning it satisfies the hypotheses of Result 2 . Since $S$ is combinatorial, we can now construct a bijection from $S$ onto $T$.

Result 4 ([6, Proposition 1.6]). If $S$ is combinatorial, for each $x$ in $S$ there is a unique element $y$ of $T$ such that $\langle x\rangle \Phi=\langle y\rangle,\left(x x^{-1}\right) \phi_{E}=y y^{-1}$ and $\left(x^{-1} x\right) \phi_{E}=y^{-1} y$.

We may now define $\phi: S \rightarrow T$ by letting $x \phi$ be the unique element $y$ as above. Properties of $\phi$ are given in the next result.

Result 5 ([6, Proposition 1.7]). If $S$ is combinatorial then so is $T$, and $\phi$ is a one-toone map of $S$ onto $T$ which extends $\phi_{E}$. Further, $\phi$ and $\phi^{-1}$ are $R$-and L-preserving. If $\theta$ is a homorphism of $S$ onto $T$ which induces $\Phi$, then $\theta=\phi$.

We can now show that $\phi$ preserves the kernel of $\sigma, K_{s}$, in modular inverse semigroups. Throughout the remainder of this paper we will assume that $S$ is not a group.

Theorem 6. If $S$ is a simple modular inverse semigroup, then $K_{S} \Phi=K_{T}$.

Proof. Take any $u \in K_{T}$. If $u \in E_{T}$, then $u \in E_{S} \Phi \subseteq K_{S} \Phi$. So assume $u \notin E_{T}$. Now $u=x \phi$ for some $x \in S$ by Result 5, and from Result 3, $u u^{-1} \| u^{-1} u$. It follows that $\left(x x^{-1}\right) \phi \|\left(x^{-1} x\right) \phi$ by Result 4 , and since $\phi$ is an isomorphism on $E_{S}, x x^{-1} \| x^{-1} x$. Applying Result 3 again $x \in K_{S}$ yielding $K_{T} \subseteq K_{S} \Phi$.

Similarly, $K_{S} \subseteq K_{T} \Phi^{-1}$, and the proof is complete. 
In a simple modular inverse semigroup $S$, every element $x$ not in $K_{S}$ is either strictly right or left regular, and therefore generates a bicyclic subsemigroup. Thus properties of $L(B)$ for the bicyclic semigroup $B$ are bound to play an important role.

Result $7([5,6])$. The bicyclic semigroup is strongly determined by its lattice of inverse subsemigroups. In fact every lattice isomorphism of $B$ onto $T$ is induced by a unique isomorphism of $B$ onto $T$.

If $S$ is a simple modular inverse semigroup and $x$ is an element not in $K_{S}$, then $\Phi$ restricted to $\langle x\rangle$ is a lattice isomorphism of $\langle x\rangle$ onto $\langle x \phi\rangle$, which from Results 4,5 and 7 is induced by $\phi$, and $\phi$ must be the isomorphism from $\langle x\rangle$ onto $\langle x \phi\rangle$. Note that Result 4 shows that an element $x$ is strictly right [left] regular if and only if $x \phi$ is.

The following generalization of Lemma 4.6 of [5] will be useful.

Lemma 8. Let $S$ be a simple modular inverse semigroup. (i) If $a$ is a strictly right [left] regular element of $S$, then so is ea for every idempotent $e \leqq a a^{-1}$. (ii) If aøb for $a, b \in s$, then $a$ is strictly right $[$ left $]$ regular if and only if $b$ is.

Proof. (i) Take any strictly right regular element $a$ of $S$ and any idempotent $e<a a^{-1}$. Then $a \notin K_{S}$ by Result 3 and so also $e a \notin K_{s}$. Clearly eaRe. Since $E_{S}$ is Archimedean, $a^{-n} a^{n}<e$ for some $n>0$, whence $a^{-n} e a^{n} \leqq a^{-n} a^{n}<e$. By way of contradiction, assume $e a$ is left regular; that is, $a^{-1} e a=(e a)^{-1}(e a)>e$. Then $a^{-2} e a^{2}=$ $a^{-1}\left(a^{-1} e a\right) a>a^{-1} e a>e$, and by induction, $a^{-n} e a^{n}>e$ which is impossible. Thus $e a$ is strictly right regular. The proof for strictly left regular is similar.

(ii) Take $a, b \in S$ with $a \sigma b$ and suppose $a$ is strictly right regular. Now $a \notin K_{S}=\operatorname{ker} \sigma$ so also $b \notin K_{S}$, hence $b$ must be strictly right or left regular. Since $a \sigma b$ there is an $e \in E_{S}$ such that $e a=e b$, and without loss of generality we may take $e \leqq a a^{-1}$. By (i) above, $e a$ is strictly right regular and thus so is $e b$. Again using (i), $b$ cannot be strictly left regular, so the desired result follows.

Before proceeding further we will need several technical results.

Result 9 ([2, Proposition 2.9]). If $S$ is a simple modular inverse semigroup, then $S / \sigma$ is a torsion-free abelian group.

Result 10 ([2, Lemma 1.8]). Let $S$ be an inverse semigroup and $b \in S, b \notin E_{S}$. If $x$ is a nonidempotent in $\left\langle E_{S}, b\right\rangle$ then $x=x x^{-1} b^{n}$ for some non-zero integer $n$.

Lemma 11. If $x$ and $z$ are strictly right regular elements of a simple modular inverse semigroup $S$ and $\langle x\rangle \sigma=\langle z\rangle \sigma$, then $x \sigma z$.

Proof. We must have $x \sigma w$ for some $w \in\langle z\rangle \subseteq\left\langle E_{S}, Z\right\rangle$. By Result $10, w=w w^{-1} z^{n}$ for some non-zero integer $n$, so $x \sigma z^{n}$. Similarly, $z \sigma x^{m}$ for some $m \neq 0$, and it follows that $x \sigma x^{m n}$. Since $x \notin K_{S},\langle x\rangle$ is torsion-free by Result 9 , so $x \sigma=(x \sigma)^{m n}$ implies $m n=1$, whence $n= \pm 1$. This gives us $x \sigma z^{ \pm 1}$, but since $z^{-1}$ is strictly left regular, we cannot have $x \sigma z^{-1}$ by Lemma 8 (ii). It now follows that $x \sigma z$. 
Lemma 12. If $S$ is a simple modular inverse semigroup and $z=f x$ where $x, z \in S \backslash K_{S}$, then $(\langle z\rangle \Phi) \sigma=(\langle x\rangle \Phi) \sigma$.

Proof. From Results 4 and $5, c=z \phi$ and $a=x \phi$ are such that $c, a \in T \backslash K_{T}$. Now since $z=f x,\langle z\rangle \subseteq\left\langle E_{S}, x\right\rangle=E_{s} \vee\langle x\rangle$, so $\langle c\rangle=\langle z\rangle \Phi \subseteq E_{S} \Phi \vee\langle x\rangle \Phi=E_{T} \vee\langle a\rangle=\left\langle E_{T}, a\right\rangle$. Thus by Result $10, c=c c^{-1} a^{n}$, for some non-zero $n$, and hence $c \sigma a^{n}$. Let $y=$ $\left(a^{n}\right) \phi^{-1} \in\left\langle a^{n}\right\rangle \Phi^{-1} \subseteq\langle x\rangle$. Now $\langle y\rangle \subseteq\langle x\rangle$ and $\langle z\rangle=\langle c\rangle \Phi^{-1} \subseteq\left\langle E_{T}, a^{n}\right\rangle \Phi^{-1}=E_{T} \Phi^{-1}=$ $E_{T} \Phi^{-1} \vee\left\langle a^{n}\right\rangle \Phi^{-1}=\left\langle E_{S}, y\right\rangle$. Applying Result 10 again, $z=z z^{-1} y^{m}$, for some non-zero $m$, and so $x \sigma z \sigma y^{m}$.

Since the mapping $\phi$ restricted to $\langle x\rangle$ is an isomorphism onto $\langle a\rangle, y=\left(a^{n}\right) \phi^{-1}=$ $\left(a \phi^{-1}\right)^{n}=x^{n}$, and combining this with $x \sigma y^{m}$ gives $x \sigma x^{m n}$. We know that $\langle x \sigma\rangle$ is torsion-free since $x \notin K_{s}$, so as in the previous proof, $n= \pm 1$. From this we get $a=a^{ \pm 1}$, so $\left\langle a^{n}\right\rangle=\langle a\rangle$, and finally $(\langle z\rangle \Phi) \sigma=\langle c\rangle \sigma=\left\langle a^{n}\right\rangle \sigma=\langle a\rangle \sigma=(\langle x\rangle \Phi) \sigma$ as desired.

Notice that if $a \sigma b$ in a simple modular inverse semigroup $S$, and $a, b \notin K_{S}$, then $e a=e b \notin K_{S}$ for some idempotent $e$. The above lemma shows that $(\langle e a\rangle \Phi) \sigma=(\langle a\rangle \Phi) \sigma=$ $(\langle b\rangle \Phi) \sigma$, or equivalently, $\langle a \phi\rangle \sigma=\langle b \phi\rangle \sigma$. Applying Lemma 11, $(a \phi) \sigma(b \phi)$. Thus we have proved:

Corollary 13. If $S$ is a simple modular inverse semigroup and $a, b \in S$, then arb implies $(a \phi) \sigma(b \phi)$.

We know that $\phi$ is $R$-, $L$ - and $\sigma$-preserving on a simple modular inverse semigroup $S$, and that $\phi$ is an isomorphism on $E_{S}$. These results will be crucial in proving our main theorems.

Theorem 14. If $S$ is is a simple modular inverse semigroup, then $\phi$ is a homomorphism.

Proof. Take any $a, b \in S$ and put $f=\left(a^{-1} a\right)\left(b b^{-1}\right)$. Then $(a b) R(a f) L(f) R(f b) L(a b)$, and since $\phi$ is $R$ - and $L$-preserving.

$$
(a b) \phi R(a f) \phi L(f \phi) R(f b) \phi L(a b) \phi
$$

By a result of Miller and Clifford [7],

$$
(a b) \phi H(a f) \phi(f b) \phi
$$

and since $T$ is combinatorial,

$$
(a b) \phi=(a f) \phi(f b) \phi
$$

But $(f b) \sigma b$, so $(f b) \phi \sigma(b \phi) \sigma(f \phi)(b \phi)$, and since $\phi$ is an isomorphism on $E_{S}$ and is $R$ preserving, $f \phi \leqq\left(b b^{-1}\right) \phi=(b \phi)(b \phi)^{-1}$, so that

$$
((f b) \phi,(f \phi)(b \phi)) \in R \cap \sigma .
$$


We will show that $(f b) \phi=(f \phi)(b \phi)$. To do this we now consider two cases.

Case I. The element $b$ is in $K_{s}$. Then $f b \in K_{S}, b \phi \in K_{T}$, and so $(f b) \phi$ and $(f \phi)(b \phi)$ are in $K_{T}$. Suppose $(f b) \phi=f \phi$.

Notice that if $x$ is any element of $K_{S}$ (or $K_{T}$ ), Result 3 tells us that $x^{2}=x^{3}$. From this we can see that $x^{2}$ is idempotent, for

$$
\left(x^{2}\right)^{2}=x^{3} x=x^{2} x=x^{3}=x^{2}
$$

Also note that

$$
\begin{gathered}
x^{2} x^{-1}=x^{-2} x^{-1}=x^{-3}=x^{3}=x^{2}, \text { and thus } \\
x^{-1} x^{2} x^{-1}=\left(x^{-1} x\right)\left(x x^{-1}\right)=x^{2} .
\end{gathered}
$$

Applying this to $b$ and $b \phi$ we have

$$
\begin{aligned}
\left(b^{2}\right) \phi & =\left[\left(b^{-1} b\right)\left(b b^{-1}\right)\right] \phi=\left(b^{-1} b\right) \phi\left(b b^{-1}\right) \phi \\
& =(b \phi)^{-1}(b \phi)(b \phi)(b \phi)^{-1}=(b \phi)^{-1}(b \phi)^{2}(b \phi)^{-1}=(b \phi)^{2} .
\end{aligned}
$$

We previously assumed that $(f b)=f \phi$, and since $\phi$ is a bijection, $f b=f$, and we can calculate $f b^{2}=f b=f$. Therefore $(f \phi)(b \phi)=\left(f b^{2}\right) \phi(b \phi)=(f \phi)\left(b^{2} \phi\right)(b \phi)=(f \phi)(b \phi)^{2}(b \phi)=$ $(f \phi)(b \phi)^{3} \in E_{T}$ so that $(f \phi)(b \phi)=(f b) \phi=f \phi$, as required.

If we assume rather that $(f \phi)(b \phi)=f \phi$, then let $e=f \phi$ and $c=b \phi$. Then $(f \phi)(b \phi)=$ $f \phi$ becomes $e c=e$, and (2) is

$$
\left((e c) \phi^{-1},\left(e \phi^{-1}\right)\left(c \phi^{-1}\right)\right) \in R \cap \sigma
$$

since $\phi^{-1}$ is $R$ - and $\sigma$-preserving. Thus the same argument as above, applied to $\phi^{-1}$, yields $(e c) \phi^{-1}=\left(e \phi^{-1}\right)\left(c \phi^{-1}\right)$, in other words $f=f b$ as before. Thus, $(f b) \phi=(f \phi)(b \phi)$ in this case also.

Since, by Result $3\left|K_{T} \cap R_{f \phi}\right| \leqq 2$, if neither $(f b) \phi$ nor $(f \phi)(b \phi)=f \phi$, then $(f b) \phi$ and $(f \phi)(b \phi)$ must be equal.

Case II. The element $b$ is not in $K_{s}$. Let $U=\left\langle E_{s}, b\right\rangle$. Now $U \cap K_{s}=E_{s}$, for if $x$ is a nonidempotent in $\left\langle E_{S}, b\right\rangle, x=x x^{-1} b^{n}$ for some non-zero integer $n$ by Result 10 . Then the subgroup $\langle x \sigma\rangle=\left\langle b^{n} \sigma\right\rangle$ of $S / \sigma$ is nontrivial since $b \sigma \neq 1$ and $S / \sigma$ is torsion-free, and so $x \notin K_{s}$. From this it follows that $U$ is $E$-unitary. Recall that $((f b) \phi,(f \phi)(b \phi)) \in R \cap \sigma$ (in $T)$, and it easy to verify that also $((f b) \phi,(f \phi)(b \phi)) \in R_{U \phi} \cap \sigma_{U \phi}$.

By [6, Theorem 3.4] $U \phi$ is E-unitary. From [8, Proposition III.7.2], that $U \phi$ is $E$-unitary implies that $R_{U \phi} \cap \sigma_{U \phi}$ is the identical relation on $U \phi$. Hence we get get $(f b) \phi=(f \phi)(b \phi)$, as desired. A similar argument yields $(a f) \phi=(a \phi)(f \phi)$.

Now we have that (1) is equivalent to

$$
(a b) \phi=(a \phi)(f \phi)(b \phi) .
$$


But since $f=\left(a^{-1} a\right)\left(b b^{-1}\right), f \phi=\left(a^{-1} a\right) \phi\left(b b^{-1}\right) \phi=(a \phi)^{-1}(a \phi)(b \phi)(b \phi)^{-1}$, and replacing $f \phi$ with this in (3) gives $(a b) \phi=(a \phi)(b \phi)$.

Combining this last theorem with Result 5 we can now state the main result of this paper.

Theorem 15. Let $S$ be a simple modular inverse semigroup which is not a group. Then each isomorphism of $L(S)$ onto $L(T)$ is induced by a unique isomorphism of $S$ onto $T$.

\section{REFERENCES}

1. J. M. HowIE, An Introduction to Semigroup Theory (Academic Press, London, 1976).

2. K. G. Johnston and P. R. Jones, Modular inverse semigroups, J. Austral. Math. Soc. Ser. A, to appear.

3. P. R. Jones, Semimodular inverse semigroups, J. London Math. Soc. 17 (1978), 446-456.

4. P. R. Jones, Distributive inverse semigroups, J. London Math. Soc. 17 (1978), 457-466.

5. P. R. Jones, Lattice isomorphisms of distributive inverse semigroups, Quart. J. Math. Oxford 30 (1979), 301-314.

6. P. R. JoNEs, Inverse semigroups determined by their lattices of inverse subsemigroups, $J$. Austral. Math. Soc. Ser. A 30 (1981), 321-346.

7. D. D. Miller and A. H. Clifford, Regular D-classes in semigroups, Trans. Amer. Math. Soc. 82 (1956), 270-280.

8. M. Petrich, Inverse Semigroups (Wiley, New York, 1984).

9. L. N. Sevrin and A. J. Ovsyannikov, Semigroups and their subsemigroup lattices, Semigroup Forum 27 (1983), 1-154.

Department of Mathematics

College of Charleston

Charleston, SC 29424

U.S.A. 